\title{
An Evolutionary View of Collective Intelligence
}

\author{
Bengt Carlsson ${ }^{1}$ and Andreas Jacobsson ${ }^{2}$ \\ ${ }^{I}$ School of Computing, Blekinge Institute of Technology, 37179 Karlskrona, Sweden \\ ${ }^{2}$ Faculty of Technology and Society, Malmö University, 20506 Malmö, Sweden \\ bengt.carlsson@bth.se, andreas.jacobsson@mah.se
}

Keywords: Collective Intelligence, Artificial Intelligence, Evolution, Meme.

Abstract: $\quad$ Based on the question "How can people and computers be connected so that - collectively - they act more intelligently than any individuals, groups, or computers have ever done before?" we propose an evolutionary approach. From this point of view, there are of course fundamental differences between man and machine. Where one is artificial, the other is natural, and where the computer needs to process, the brain must adapt. We propose the use of culturally inherited units, i.e., memes, for describing collective knowledge storage. Like the genes, memes have the ability to be inherited to the next generation. Genes appear independently of our society while memes are a result of our cultural development. The concept of collective intelligence may involve a new kind of meme, entirely emerging within the intersection between man and machine, i.e., outside the scope of human control. The challenge is to model this behavior without overriding constraints within basic evolutionary vs. machine settings.

\section{INTRODUCTION}

Artificial intelligence touches upon a popular philosophical question, stemming from the early days of computer science; how much human intelligence can actually be emulated on a computer? Initially, this was a matter of humans vs. machines, or to be specific; the entire human species on one side and one, often presumed to be gigantic, computer with ultimate superior intelligence capabilities on the other.

In some areas, e.g., board games and quiz contests, the computer has proved to be at least as clever as man. In the American TV game Jeopardy, a single super computer, named Watson, succeeded in winning over several human grandmasters. The performance was impressive because the questions in part consisted of puns, irony, and other sorts of information that is difficult to interpret, calculate, and perceive by a computer. The engineers behind Watson had to use a combination of a huge database (also referred to as a knowledge base) and a rule based machine-learning system in order to estimate the probability of providing a correct response.

Does this mean that the intelligence of a computer, such as Watson, can be seen as the equivalent with that of a human being? This question dates back to the early 1950's when Alan Turing introduced what would later become known as the Turing Test (1956). Briefly, in the Turing Test, a human judge engages in a natural language conversation with a human and a machine designed to generate performance indistinguishable from that of a human being. All participants are separated from one another. If the judge cannot reliably tell the machine from the human, the machine is said to have passed the test. The test does not check the ability to give the correct answer; it checks how closely the answer resembles typical human answers. So far, none have succeeded in this test, apart from the (too) limited domains or applications of, for instance, a Chess play (see the current status at http://www.loebner.net/Prizef/loebner-prize.html).

Another related topic is the Fifth Generation Computer Systems project (FGCS), which was an effort spanning hundreds of million dollars, in which information was massively parallel-processed using logical programming languages (Fuchi, 1984). A mainframe-like environment was created where a large number of processors collaborated in order to achieve a hitherto unprecedented processing power, and where "smart" programming analyses were performed. In the early 1980's, FGCS was virtually the dream of artificial intelligence. Even if this technology is outdated - at the time, Internet had not yet received its breakthrough, and today's powerful multi-processor machines were still distant - it must be said that the project was far ahead of its time.

In another important artificial intelligence initiative, Cyc (Randall and Lenat 1982), from the word Encyclopedia, an attempt was made to assemble a comprehensive ontology and knowledge base of everyday common sense knowledge. The idea was to enter as much information as possible in a computerized storage capacity, which would establish a common vocabulary for automatic 
reasoning. The goal was to enable artificial intelligence applications to perform human-like reasoning, or even to make a computer smarter than a human being. Cyc has been considered to be a controversial endeavor, and has suffered its share of criticism. Among many things, a large number of gaps in not only the ontology of ordinary objects, but an almost complete lack of relevant assertions describing such objects, has been contributing to the increasingly fading interest of Cyc.

During the first decade of the new millennium, the debate whether or not to achieve artificial intelligence that can be measured against human intelligence, is not about a single, or even a few, super computers. It is more a question of what can be done by collective, collaborative computing efforts. Thus, can, and if so; how, a collective intelligence arise through the interaction between men and machines? The question is whether or not the appropriate preconditions for this is the Internet with all its connections, i.e., men to men, men to machines, and machines to machines.

The article is organized as follows. First we discuss the evolution of user-driven collaboration on the Web with respect to a common platform for artificial intelligence. Next, we compare computer intelligence to the human brain. Collective intelligence with respect to men and machines are then discussed. Finally, the concept of memes is debated, and the paper is concluded with some observations and points for further discussion.

\section{COMPUTERS WITH COLLECTIVE INTELLIGENCE}

So, with the introduction of the commercial Internet, i.e., the World Wide Web, or simply the Web, in the mid 1990's, companies realized that the content in this environment could actually be developed by the users, i.e., the customers, themselves. Customers shared reviews on items that they have purchased, software manufacturers used customers as product support in the development phase, and cooperating users built an entire encyclopedia of knowledge. Google became one of the world's most successful companies by utilizing Web search content provided by the users, and Facebook concurred the social side of the Web by providing means to link people, and their personal information, together.

In the book "We are the Web" (2005), Kelly described this development. The massive input of information provided by the users into the World Wide Web was referred to as "The Machine", i.e., a large artificial brain, with a capacity comparable to a human brain. The Web, like the brain, has hundreds of billions of neurons (or Web pages), joined by multiple synapses (or hyperlinks), and in turn made up of billions of transistors available in our regular computers.

Together, said Kelly, this structure connected to sensors in virtually all electronic equipment, will have sufficient complexity to independently start to learn things. Smart algorithms in combination with a global database will be able to register (in theory) almost unlimited amounts of information that can be processed in the universal cloud of computers. Every time a user clicks on a link, a node becomes a little bit better. As Kelly concluded (2005):

"We will live inside the Machine and, by that, head towards superior intelligence."

Gelernter (1993) described a Mirror World where people would interact and transact with digital representations of the real world, something as:

"A true-to-life mirror image trapped inside a computer. [...] The whole point of a mirror world is that it is wired in real time and place - it is supposed to mirror reality rather than being a parallel reality or cyber world."

Put another way, reality is mirrored in the eyes of the user, e.g., composed by the billions and billions of "hits" that passes through, e.g., Google's search engine. This engine, in turn, can be described as an instance of evolutionary development where capabilities gradually, almost imperceptibly, are improved; our spelling mistakes are corrected, the engine determines whether personal names or places are used, suggests translations, etc. As such, it determines the connection between multiple keywords and combine different media and languages.

Among many things, Google improves its search engine by analyzing short clicks, i.e., those of users who did not find what they were looking for immediately. Google also tries to find patterns in the massive amounts of data that the users feed to the search engine. This is achieved by using machinelearning techniques, training algorithms, and ideas from control and gaming theory. Large amounts of text string examples are analyzed, and in this case; size matters - according to Google a doubling of the sample size means an improvement of 0.5 percent (Levy, 2011).

A half percent may not sound much, but this small portion indicates something much larger than finding a more efficient search algorithm. If it is possible to capture, not only the syntax, but also the semantics, this half percentage may represent an important step towards, what Tim Berners-Lee (2000) described as a Web of data that can be processed directly and indirectly by machines, which is an important step towards artificial intelligence. Ultimately, this can enable us to reach beyond human intelligence, i.e., computers as the most cleaver "beings" on earth. But is this realistic? What 
about the constraints that are built into the silicon cover, outmost depending on the binaries of a simple 0 and 1, conveyed through a programming language? It must be pointed out that this question of course only is valid, presuming that all computers are digital. Much effort is spent on wetware computers, quantum computers, and chemical computers, but that is a discussion outside the scope of this paper. Before, we can take on such a challenge; we must first investigate how a computer's memory and processing differs from a human brain.

\section{COMPUTER INTELLIGENCE VS. THE HUMAN BRAIN}

Obviously, the brain is not a search engine; there are significant differences in how information is both stored and processed in a brain compared to a computerized setting. While a huge storage capacity, where data is never forgotten, is a benefit for the computer, the opposite can be said for the brain. The information processing within a computer changes the flow of binaries, where the brain alters the anatomy resulting in a whole chain of processes that need to be activated.

To clarify this, four different topics are introduced in this section: interaction, memory, processing, and environment.

\subsection{Interaction}

When submitting a search query on Google's search engine, we often do not know precisely what we are looking for, but we can extract such an answer by refining the search (based on the information on the first search result). We ask the question, and the program, in this case Google, responds. Computers have become better and better at providing users with answers, but only to the questions that a programming language is able to return an answer in. Humans, on the other hand, rely on sense rather than on calculation, i.e., we give answers without always realizing the underlying problem. Imagine now that the process is reversed: the computer asks a human to give the answer to a question set by a program.

Google's search engine, with its modified PageRank algorithm, is a system that investigates and saves the judgments a person makes when he/she links to or looks at a specific page (Levy 2011). What the user clicks on depends on how well the search phrase that the human fed into the system match the information, which the human is in search for, and that Google suggests. The system learns both from experienced users (who are skilled in formulating search phrases and identifying matches) and inexperienced users (who are incapable or unused in formulating search phrases and locating matches). So, a person has indirectly answered the question regarding the importance of a specific Web site (in relation to other Web sites), and thus a small part of "human intelligence" is integrated into the artificial computer brain of Google's search engine. The rest is actually rather trivial; all searches are combined, and the result gets increasingly more adequate.

The vision of the intelligent interconnected Web is based on what we as humans do inside the World Wide Computer through both professional and personal relationships. We are nodes on the Web where intelligence becomes a part of the global computer with its ubiquitous "intelligence" embedded in software code, databases, and microchips. The intelligence is in the eye of the beholder; it is actually about how the interaction with the Internet-connected computer will affect our lives.

\subsection{Memories}

Digitally stored information, or let us call it memory, is becoming easier to access. Google's ambition is, by using smart algorithms and massive server power, to process all stored information in order to complement and surpass human capacity. By releasing unnecessary memory storage in our brain, humans may use their brains for better and more creative purposes. Instead of recalling information, memory can be stored digitally and recreated when needed. In the end, according to these visionaries, our memory capacity increases by paving the way to a more valuable, more human, processing of information. In this context, the crucial question to ask is how the human memory differs from the computer's information storage.

A full backup, i.e., conducting a complete copy of data, is a common activity between computers. This is the simplistic equivalence to a transplant of human memory to a computer; transfer all original contents of a computer to an exact replica.

However, other things are also included in the transmission. An initially systematic storage becomes increasingly fragmented, and hidden among the accessible information is undefined information, e.g., deleted and cashed files. This is an equivalent to "age ailments" among humans demonstrated by computer performance degradation, memory insufficiencies, hard drive crashes, etc.

To put it mildly, it is not a simple task to transfer information between humans and computers; we use our memory in the interaction with the environment where there is no storage of "death information". This is a major difference in the storage structure between a computer and a human, i.e., where the computer needs to process, the brain must adapt. 
Human memories must be adapted to the evolutionary development, and to be a resource for making the right decisions in a dynamic and changing surrounding.

Man's own combination of being able to understand a complex world, combined with an imperfect memory, is probably a really excellent adaptation to our environment. Contrary, imagine that we remembered everything we have done in the past. If our brain was more like a computer, everything should be recorded; visual inputs, sounds, and smells. An ordinary walk would result in gigabytes of stored information; fixation points for the eye, a shoe touching the ground, and all meta-information in the form of the thoughts we thought during the walk. Instead, we need to pick out important information, evaluate, and restructure the old information into something new, and sort out the non-essentials. Our intelligence is based more on being able to dismiss information rather than to store only the useful information.

So, despite the computer being more powerful when it comes to making decisions, it has a weakness in the way memories and information is stored and processed. This is also supported by what we know about the brain's memory storage (Kandel 2006).

\subsection{Processing}

As new computer materials, processors, algorithms, etc., are introduced, the computer is increasingly often compared to a human brain. A deficiency in this analogy is that all the processed data are located in main memory or storage memory of the computer, and it thus looks the same way the next time it is used. This is hardly the case with human memory. To re-evaluate and restructure old information, in humanlike ways, are tasks that are not plausible for a computer. So how does the brain's own process work?

Our brain consists of a short-term memory, which holds a continuous throughput of information, and a long-term memory that holds the capacity to maintain information for a lifetime. These two memory capacities represent two diverse biological processes. Short-term memory strengthens or weakens existing connections in the brain, through synapses, and between brain cells. Long-term memory alters the anatomy of the brain; new synapses are formed, which require the production of proteins that in turn need to activate dormant genes (LeDoux 2002). A whole chain of processes has to be activated in the formation of new synapses. This is a time consuming process separated from the distributed storage model in a computer or a cloud setting.

Instead of just storing bits and bytes, human brains have a continuous, undetermined, organic growth. The brain continues to process information long after it has been received and the quality of memory depends on this outcome. The human brain thus holds the capacity of a vivid memory instead of the "dead" artificial computer equivalent. Unlike a computer, when a long-term memory is returned to the working memory it looks different to the initially stored data. A new context is thus formed in a constant process of renewal (Carr 2010).

\subsection{Environment}

If we see the human brain as being involved in an evolutionary process, this means that we store things we find useful, and thus reduce the amount of information that we perceive as un-useful. All new branches and rearrangements of memory routes in the brain are developed to make us more prepared to meet both external dangers and to take advantage of opportunities. Instead of processing stored data from other stored data, the brain adapts to the surrounding environment and reacts accordingly. Despite the otherwise enormous capacities of computer storage, in this area it cannot compete with human capabilities. Why? The computer does not evolve according to evolutionary principles.

This inconsistency between the human brain and its computerized counterpart is present during the human computer interaction. It takes hours to transmit information to the long-term memory in the brain, which also may require repetition, i.e., learning time to transfer knowledge into long-term memory. In addition, the brain is not intended for other tasks as long-term memory elastically expands and contracts, i.e., due to adjustment in the number of synapses.

Humans do not limit - but reinforce - the mental strength when new information is stored in the longterm memory (where the computationally stored information is more limited). If we let a computer store and provide our memories with a stream of competing messages, we get an overload of working memory, i.e., we get quantity instead of quality. This means that our frontal lobe cannot focus on any particular task. In turn, this means that the hippocampus, a part of the limbic system in the brain responsible for the formation of new memories, is unable to consolidate information and therefore cannot transfer external stimuli into longterm memory. Using the computer approach our minds are trained to be confused; information is processed quickly and efficiently, but without sustained attention. Instead, when using the evolutionary approach, the brain becomes skilled at forgetting, but unapt to remember, i.e., it gets more idle-headed to think instead of relying too much on computers' artificial memory. 


\section{COLLECTIVE INTELLIGENCE}

The Center for Collective Intelligence at MIT (http://cci.mit.edu/) asks the question: "How can people and computers be connected so that collectively - they act more intelligently than any individuals, groups, or computers have ever done before?" This is an ambitious project that introduces the need for new programming metaphors, e.g., creating social operating systems, defining new programming languages, and promulgating new software engineering skills (Bernstein et al. 2012). This may also be a too optimistic project since it focuses on reciprocation between man and computer at an equal footing, or on an offering beyond human collective intelligence.

\subsection{Computers}

Computers are useful when it comes to supplying stored information, and humans are good at processing intellectual impressions and communicating different emotions. A computer vision on future opportunities suffers from two serious flaws: overconfidence and an inadequate approach to technology. Nothing indicates that the computer or the data cloud it is connected to in the future will store and process memories in a more biological way, i.e., humans will continue to be responsible of the more intuitive intelligent choices. As a tool, or an intellectual sidekick, the computer has an enormous importance, but we should not overestimate what it actually does. If we limit ourselves to what the computer can do, we restrict ourselves. It is this distinction between an extremely effective tool and the way we act towards the basic biological conditions that is a truly exciting challenge of the digital revolution.

\subsection{People}

Human progress and technological innovation can fundamentally change our lives. In evolutionary terms, this course of events takes much longer time than the Internet has been around. The mental change, our way of living and using computers, can be developed much faster. The critical issue is not to keep all the elements we now associate with our cognitive abilities to ourselves. The intelligence and technological progress are instead based on humans being extremely adaptable to new environments, i.e., using all available tools, also computerized artifacts, for collective intelligence.

\subsection{Collective Intelligence}

Basically, we contrast, instead of compare, human with computer skills, as they are inherently different from one another. We need to combine humans and computers in a more indirect way; we call this Cause and Effect.

The cause is the humans living, i.e., reacting, in a natural environment with a vivid memory adapting to evolutionary principles. The effect is (solitary) computers calculating possible options and simulating preferred outcomes. The synthesis may be called collective intelligence connected to individuals, groups, and interlinked computers.

As seen in Section 3, there are three main skills separating humans from computers, namely: memories, processing, and environment. Together they form a dividing line not possible to override for a computer singlehandedly. The remaining skill, interaction, is in the area of progress - so how do we evaluate this development compared to other major transitions in human evolution?

\section{DISCUSSION}

In their book "The Major Transitions in Evolution" (1995), Maynard Smith and Szathmáry mention the origin of language as the last transition that had a genetic basis. The invention of writing and electronically store or process data are major transitions without the genetics involved, i.e., they may have a much faster growth.

Knowledge storage includes our ability to both store and process knowledge. While this is absolutely necessary for our cultural development, it is also an area where our evolutionary background is an important feature. A meme is a culturally inherited unit, comparable to genes, which has its own survival and reproduction in a cultural environment (Dawkins 1982). Like the genes, memes have the ability to be inherited to the next generation. Genes appear independently of our society while memes are a result of our cultural development.

A connection to the memes is the large-scale collection of keywords that Google uses. A spelling that is based on selecting the spelling of a word that occurs the most frequently usually yields the correct word, i.e., spreading the correct spelling of words at the expense of the misspelled words. New ideas, fashion trends, and so on, can be described as more complex memes that can increase or decrease within the Google meme-pool.

Google's vision is to make the search engine into a system that is as smart, or smarter, than man. But the intelligence is in the eyes of the beholder, it is the person behind the keyboard that makes the informed decisions. Unlike the computer, humans live in the real world where decisions are assessed directly, and not through a meta-level of externally achieved decisions. 
Having said this, the available meme pool may virtually provide information on the real world, both locally and on a global scale. We may even track how successful new memes arise, the extinction of less successful competitors, and how stable a meme is over a longer period. This will be an indicator of how robust the society is, or an indicator of changes in progress.

Conceptually, collective intelligence is judged by humans originally serving the meme pool with new concepts, computers processing the information and the overall Web, which is storing, developing, and merging memes. In a feedback loop memes are not only stored, within humans, but within computers and (indirectly) the Web.

So, nothing in the discussion above contradicts the concept of collective intelligence. We may speculate on a new kind of meme entirely emerging within the intersection between man and machine, i.e., outside the scope of individual control. This may lead to more independent decision-making, i.e., computers may act more intelligently with respect to humans, but it is not the same as replacing human reasoning with a self-replicating artificial entity.

\section{CONCLUSION}

We propose the use of an evolutionary setting when analyzing the question of how people and computers can be connected so that - collectively - they act more intelligently than any individuals, groups, or computers have ever done before. Basically, from an evolutionary point of view, the computer processes where the brain adapts, i.e., in this respect, there is a fundamental difference between man and machine. Knowledge storage is represented by memes, culturally inherited units having a much faster growth. The concept of collective intelligence may involve a new kind of meme entirely emerging within the intersection between man and machine, i.e., outside the scope of human control. This development needs to be progressed within the evolution vs. machine constraints, i.e., human reasoning is not equalized by a self-replicating artificial entity.

So, collective intelligence is judged by humans, and processed by computers while allowing the overall Web to store, develop, and merge memes. The question of acting more intelligently than any individuals, groups, or computers have ever done before may therefore end up being an issue of how robust the society is, or an issue of changes in progress.

\section{REFERENCES}

Berners-Lee, T., 2000. Weaving the Web: The Original Design and Ultimate Destiny of the World Wide Web, HarperBusiness.

Bernstein, A., Klein, M., and Malone, T.W., 2012. Programming the global brain, Communications of the ACM, vol55, No 05.

Carr, N., 2010. The Shallows - How the Internet is Changing the Way We Think, Read and Remember, Atlantic Books.

Dawkins, R. 1982. The Extended Phenotype, W.H. Freeman and Company.

Fuchi, K., 1984. Revisiting Original Philosophy of Fifth Generation Computer Systems Project, In Proceedings of FGCS.

Gelernter, D., 1993. Mirror Worlds: or the Day Software Puts the Universe in a Shoebox...How It Will Happen and What It Will Mean, Oxford University Press.

Kelly, K., 2005. We are the Web, Wired, Issue Aug. 13.

Kandel, E.,R., 2006. In Search of Memory: the Emergence of a New Science of Mind, Norton.

LeDoux, J., 2002. Synaptic Self: How Our Brains Become Who We Are, Penguin.

Levy, S., 2011. In the Plex-How Google Thinks, Works, and Shapes Our Lives, Simon \& Schuster.

Maynard Smith, J., and Szathmáry, E., 1995, The Major Transitions in Evolutions, W.H. Freeman and company.

Turing, A., 2009 (1956). Computing Machinery and Intelligence, in ed. Epstein, R., Roberts, G., and Beber, G., Parsing the Turing Test-Philosophical and Methodological Issues in the Quest for the Thinking Computer, Springer Verlag.

Randall, D., and Lenat, D.B., 1982. Knowledge-Based Systems in Artificial Intelligence, McGraw-Hill. 